\title{
The efficacy and safety of oral Tamsulosin controlled absorption system (OCAS) for the treatment of lower urinary tract symptoms due to bladder outlet obstruction associated with benign prostatic hyperplasia: An open-label preliminary study
}

\author{
Bannakij Lojanapiwat, Sompol Permpongkosol \\ Division of Urology, Department of Surgery (BL), Chiangmai University, Chiangmai and Division \\ of Urology, Department of Surgery (SP), Ramathibodi hospital, Bangkok, Thailand
}

\begin{abstract}
Aims: Tamsulosin, a superselective subtype alpha 1a and $1 \mathrm{~d}$ blocker, is used for the treatment of male Lower Urinary Tract Symptoms (LUTS) commonly caused by Benign Prostatic Hyperplasia (BPH). This prospective study evaluated the efficacy and safety of a new formulation, Tamsulosin OCAS ${ }^{\circledR}$ (Oral Controlled Absorption System), for LUTS associated with BPH in Thai patients.

Materials and Methods: Fifty one patients over 40 years old with complaints of LUTS associated with BPH were recruited. Patients received an 8 week course of once daily $0.4 \mathrm{mg}$ tamsulosin OCAS ${ }^{\circledR}$, and were followed up at 2 (visit 3), 4 (visit 4) and 8 (visit 5) weeks post-treatment. At each visit, patients were assessed using the International Prostate Symptom Score (IPSS), Nocturia Quality of Life (N-QoL) Questionnaire, QoL Assessment Index (IPSS-QoL), and International Index of Erectile Function (IIEF). The primary outcome was efficacy of Tamsulosin. The secondary outcomes included change in the mean number of nocturia episodes, hours of undisturbed sleep (HUS) and uroflowmetry measurements.

Results: Total IPSS significantly decreased at week 8 from baseline (from 19.52 to $6.08 ; \mathrm{p}<0.001$ ). Similarly, the voiding and storage subscores of IPSS also continued to improve significantly starting from the second and third visits, respectively ( $p<0.001$ versus baseline). The IPSS-QoL and N-QoL scores significantly improved at visit 3 through end of study. In addition, we observed significant nocturia and HUS improvement in their last clinic visit. Uroflowmetry parameters, $Q_{\max }$ and $Q_{\text {ave }}$, improved significantly at $3^{\text {rd }}$ clinic visit . Three patients experienced mild dizziness.

Conclusion: Tamsulosin OCAS ${ }^{\circledR}$ treatment led to significant improvements in LUTS, HUS and QoL in Thai patients with bladder outlet obstruction from BPH with few side effects.
\end{abstract}

Key words: prostatic hyperplasia; bladder outlet obstruction; adrenergic alpha antagonists; prostate; outcome Int Braz J Urol. 2011; 37: 468-476

\section{INTRODUCTION}

Benign Prostatic Hyperplasia (BPH) is a common cause of male Lower Urinary Tract Symptoms (LUTS). LUTS consists of voiding and storage symptoms, with the latter having a greater effect on quality of life. Nocturia, defined as important complaint patients interrupt their sleep to void (1).
Alpha blockers are the first-line of medical treatment for BPH. The advantages of the newer alpha blockers for BPH include quick improvement of symptoms and quality of life, and less side effects. Tamsulosin is a superselective subtype alpha 1a and 1d blocker used for the treatment of LUTS in BPH. Tamsulosin OCAS ${ }^{\circledR}$ (Oral Controlled Absorption System) is a new formulation of Tamsulosin which 
has consistent drug release independent of $\mathrm{pH}$. This 24-hour sustained efficacy of Tamsulosin OCAS ${ }^{\circledR}$ leads to improvement in LUTS from BPH, such as voiding symptoms and nocturia, with less side effects (2-5). Asian patients have different characteristic from the western patients, especially, in regards to body mass index (BMI) that may affect the result of the treatment. We evaluated the efficacy and safety of tamsulosin OCAS ${ }^{\circledR} 0.4 \mathrm{mg}$ in 51 Thai patients with LUTS associated with BPH over 8 weeks of treatment.

\section{MATERIALS AND METHODS}

The study was approved by the ethics committee of the Faculty of Medicine, Chiang Mai University and Ramathibodi hospital. Patients gave written informed consent before participating in the study.

\section{Patients}

Fifty-one male patients over 40 years old with complaints of LUTS associated with BPH were recruited from the outpatient departments of the study sites. LUTS associated with BPH were diagnosed by digital rectal examination or ultrasound. Prostatespecific antigen (PSA) levels were within the normal range $(<4 \mathrm{ng} / \mathrm{dL})$. All patients had a total International Prostate Symptom Score (IPSS) greater than or equal to 13, and got up interrupting sleep to void at least 2 times per night during the week prior to recruitment. The maximum urinary flow rate $\left(\mathrm{Q}_{\max }\right)$ was between 4 and $15 \mathrm{~mL} / \mathrm{s}$ with the voided urine volume greater than $120 \mathrm{~mL}$.

The exclusion criteria included (1) previous prostatectomy; (2) severe hepatic dysfunction; (3) severe renal dysfunction; (4) severe cardiovascular disorder; (5) orthostatic hypotension; (6) senile dementia; (7) other conditions which can cause voiding dysfunction such as neurogenic bladder, bladder or urethral stone, recurrent urinary tract infection, bladder cancer, prostate cancer, urethral stricture and large bladder diverticulum; (8) history of hypersensitivity or allergy to tamsulosin; (9) symptomatic urinary tract infection in the preceding month (10) nocturnal polyuria; (11) use of 5-alpha-reductase in- hibitors within the preceding 3 months; (12) use of diuretics and sleeping pills; (13) use of other pharmacologic treatments for $\mathrm{BPH}$, such as alpha blockers or plant extracts in the preceding month; (14) use of medications, such as alpha agonists, cholinergic or anticholinergic drugs, which may influence the pharmacodynamic effects of Tamsulosin.

\section{Study Design}

Figure-1 shows the design of the study. Visit 1 (week -2) evaluate all patients for eligibility for the study. Demographic data, medical history, concomitant medications and vital signs were recorded. In addition, the IPSS, IPSS-Quality of life (QoL), Nocturia-QoL (N-QoL), International Index of Erectile Function (IIEF), sleep diary measurements including uroflowmetry, and laboratory measures were also assessed before the start of treatment. Visit 2 (week 0) was the start of drug treatment. Patients who satisfied the inclusion criteria were given 0.4 mg once daily doses of Tamsulosin OCAS ${ }^{\circledR}$ (Harnal OCAS ${ }^{\circledR}$, Astellas Pharma Inc, Japan) for a period of 8 weeks. Patients were followed up at 2 (visit 3), 4 (visit 4 ) and 8 (visit 5) weeks after treatment. At each clinic visit, patients were assessed for concomitant medications, IPSS, IPSS-QoL, N-QoL, IIEF and uroflowmetry parameters.

\section{Clinical Evaluation}

During the evaluation period, the medical history (underlying diseases), height, weight and concomitant medications were recorded. Physical examination including vital signs, general examination and digital rectal examination were done. PSA and general laboratory tests, including Complete Blood Counts $(\mathrm{CBC})$, liver function tests, kidney function tests, electrolyte, blood sugar, uric acid, lactate dehydrogenase (LDH) and Gamma-GTP, were conducted. During each visit, the blood pressure and heart rate were obtained while patients were seated. Laboratory tests were repeated at the last visit (week 8).

\section{Symptom assessment IPSS questionnaires}

Patients completed the IPSS questionnaires which consist of a total of seven questions - four on voiding symptoms and three on storage 
Figure 1 - Study design.

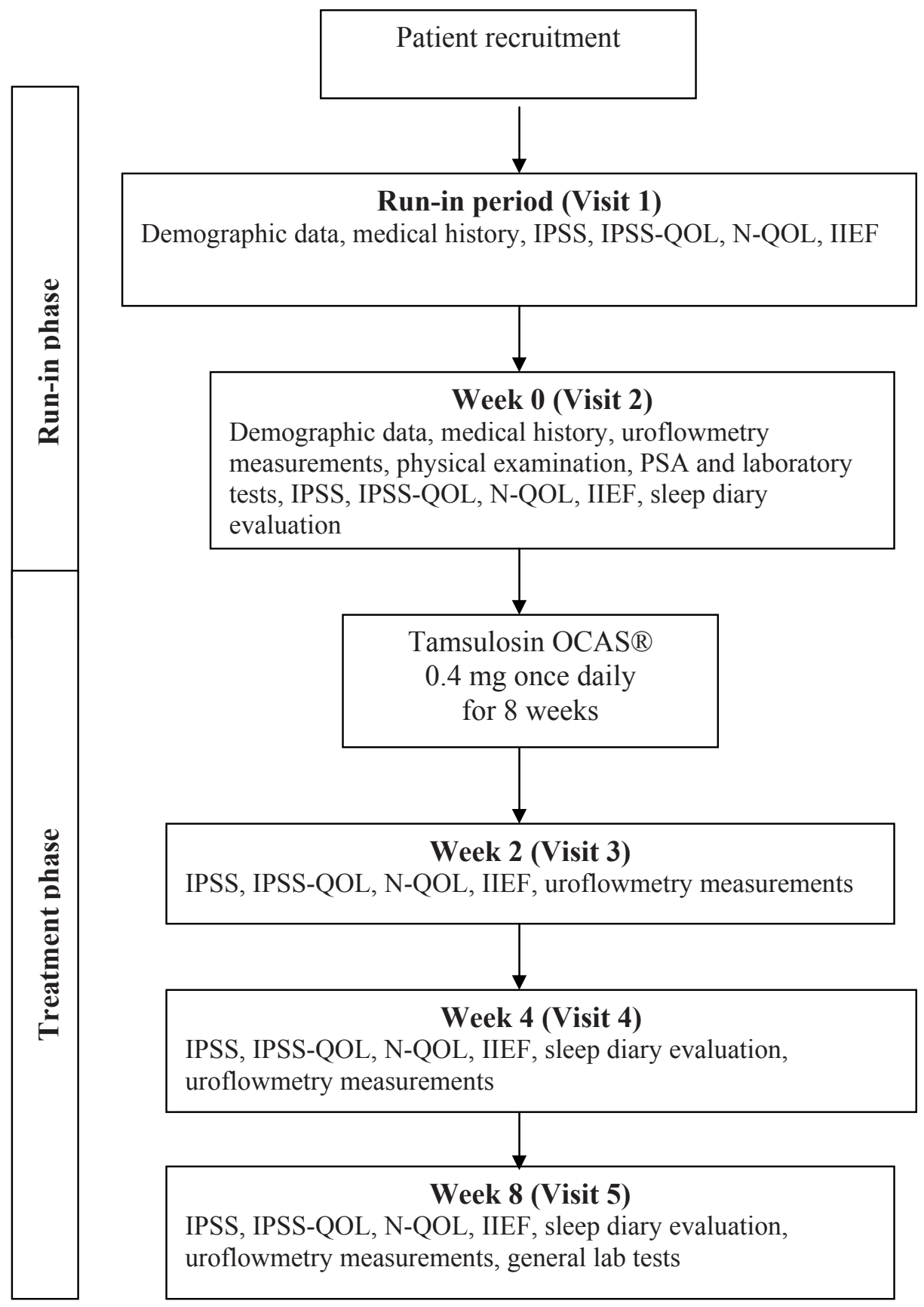

IPSS, International Prostate Symptom Score; N-QOL, Nocturia Quality of Life, IIEF, International Index of Erectile Function; OCAS, oral controlled absorption system; PSA, Prostate-specific antigen 
symptoms. The answers were graded on a five-point rating scale. The maximum values for total IPSS, voiding and storage scores are 35, 20 and 15, respectively. A high score indicates worse condition.

\section{chart)}

\section{Sleep Diary (3-day frequency/volume}

Patients received full instructions on how to complete the sleep diary and counseled on the importance of completing the diaries during their sleeping period for 3 consecutive working days (Monday to Thursday) in the week prior to the next clinic visit to provide accurate information.

Frequency, volume of each void and times going to bed and getting up were recorded in the sleep diary by the patient. Measuring jugs were used to determine the urine volumes of each void.

Sleep diaries were dispensed to patients at visits 1 (run-in), 3 (week 2), and 4 (week 4) and were evaluated at visits 2 (week 0), 4 (week 4) and 5 (week 8).

The sleep diary indicated the number of nocturia episodes and Hours of Undisturbed Sleep (HUS).

\section{Quality of life assessment \\ IPSS-QoL}

Patients answered the disease-specific QoL question contained within the IPSS questionnaire, "If you were to spend the rest of your life with your urinary condition just the way it is now, how would you feel about that?". The score for this component ranges from 0 to 6 . A high score indicates a worse condition.

\section{N-QoL}

Patients also answered the self-administered 13-item N-QoL questionnaire consisting of three domains: sleep/energy (7 questionnaires, score range from 0-28), bother/concern (5 questionnaires, score range from 0-20), and global QoL questionnaire consisting of "Overall, I would like to rate my quality of life to be?; the answer range of very good, good, fair, poor and very poor which score range from $0-4$. All questionnaires are deal with daytime energy, worry, productivity, sleep, and vitality. The total score ranged from 0 (poorest QoL) to 52 (best QoL).

\section{Sexual function assessment \\ IIEF}

Patients completed the IIEF questionnaire which consisted of 5 items based on the ability to identify the presence or absence of erectile dysfunction (ED) over the previous 6 months. The IIEF score is the sum of questions 1 to 5 . ED was classified into five severity levels, ranging from none (22-25), through severe (5-7).

\section{Uroflowmetry}

Uroflowmetric parameters, such as $\mathrm{Q}_{\max }$, average flow rate $\left(\mathrm{Q}_{\mathrm{ave}}\right)$, void volume and void time were measured at visits 2 (week 0), 3 (week 2), 4 (week 4) and 5 (week 8). For these parameters, measurements at visit 2 were considered baseline.

\section{outcomes}

\section{Evaluation of primary and secondary}

The primary outcome was efficacy of Tamsulosin OCAS ${ }^{\circledR} 0.4 \mathrm{mg}$. Efficacy was evaluated by changes in the mean values for total IPSS and IPSS subscores, IPSS-QoL, N-QoL and IIEF. Successful treatment was defined as a significant change in the mean efficacy parameters of the last clinic visit (visit 5) from baseline values (visit 1). The onset of successful treatment was the comparison between the IPSS scores (total and subscore) at baseline and each clinic follow up visit.

The secondary outcome measures were change in the mean number of nocturia episodes, HUS and uroflowmetric parameters.

In addition, the number of patients with a clinically significant response to Tamsulosin was determined, defined as those patients with a $\geq 25 \%$ decrease in total IPSS score, $a \geq 1$ point decrease in IPSS-QoL score, or $\geq 20 \%$ increase in $\mathrm{Q}_{\max }$ over baseline.

\section{Assessment of Safety}

Safety was evaluated by assessing the incidence and severity of adverse events at each clinic visit. In addition, vital signs, such as blood pressure and heart rate were also measured at every clinic visit. Laboratory parameters, including hematology, biochemistry and urinalysis, were measured at enrollment and at last visit. 


\section{Statistical analysis}

Statistical analysis was performed using SPSS 17.0 software. Analysis of variance (ANOVA) was used to assess the change in the mean of efficacy parameters and adverse events variations of each visit from the mean at baseline. For all comparisons, $\mathrm{p}<$ 0.05 was considered statistically significant.

\section{RESULTS}

Fifty-one patients were recruited into the study. Two patients did not complete the study due to development of urinary tract infection (one case) and loss of follow-up (one case). Thus, 49 patients were evaluated for treatment efficacy while all 51 patients enrolled were assessed for adverse reactions. The mean age of all patients was 63.71 years (range 48-80 years). Thirty-three patients $(67.35 \%)$ had a body mass index (BMI) between $18.5-25 \mathrm{~kg} / \mathrm{m}^{2}$ and 15 patients $(30.61 \%)$ had a BMI $>25 \mathrm{~kg} / \mathrm{m}^{2}$. The average systolic blood pressure and diastolic blood pressure at baseline were $135.97 \pm 18.33$ and $81.04 \pm 10.12 \mathrm{mmHg}$, respectively. The most common underlying disease was hypertension, which was found in 13 patients $(26 \%)$. Other underlying diseases included dyslipidaemia $(8 \%)$ and diabetes $(6 \%)$. Baseline characteristics of patients for total IPSS and IPSS subscores, IPSS-QoL, $\mathrm{N}-\mathrm{Q}$ LL and IIEF are shown in Table-1; number of nocturia episodes and HUS are shown in Table-2; and uroflowmetric parameters are shown in Table-3.

\section{Primary outcomes}

The results for the primary outcome measures are presented in Table-1. The total IPSS score significantly decreased at week 8 compared to baseline (from 19.52 to $6.08 ; \mathrm{p}<0.001$ ). A $25 \%$ improvement in total IPSS versus baseline was observed in 33 (67.35\%), 48 (97.96\%) and $49(100 \%)$ patients at visits 3 (week 2), 4 (week 4) and 5 (week 8), respectively. The IPSS subscores for both voiding and storage symptoms also decreased significantly by week 8 ( $p<0.001$ versus baseline) (Figure-2). The onset of efficacy was demonstrated at 2 weeks following treatment. The IPSS-QoL score was significantly improved at clinic visit 3 ( 2 weeks post-treatment) and throughout the end of the study (Figure-3). At least a 1-point decrease in IPSS-QoL versus baseline occurred in 39 (79.60\%), 46 (93.88\%) and 48 (97.96\%) patients at visits 3 (week 2), 4 (week 4) and 5 (week 8 ), respectively. The N-QoL scores (items (1-13)) significantly improved at visit 3 and throughout the end of the study compared with baseline (Figure-4). The IIEF score did not change throughout the study.

\section{Secondary outcomes}

The mean nocturia episodes improved significantly from baseline to week $4(\mathrm{p}<0.001)$ and week $8(p<0.001)$. Significant improvements were also found in the HUS by week $8(\mathrm{p}=0.01)$ (Table-2). Among the uroflowmetry parameters, $\mathrm{Q}_{\max }$ and $\mathrm{Q}_{\text {ave }}$ improved significantly at clinic visit 3 (Table-3). At least a $20 \%$ improvement in $\mathrm{Q}_{\max }$ versus baseline

Table 1 - Primary efficacy outcomes at baseline and after 2, 4 and 8 weeks of treatment.

\begin{tabular}{lcccc}
\hline Variable & Baseline & Visit 3 (Week 2) & Visit 4 (Week 4) & Visit 5 (Week 8) \\
\hline Total IPSS score & $19.52 \pm 4.92$ & $12.58 \pm 6.07(\mathrm{p}<0.001)^{*}$ & $7.96 \pm 4.47(\mathrm{p}<0.001)^{*}$ & $6.08 \pm 3.96(\mathrm{p}<0.001)^{*}$ \\
- Voiding score & $12.38 \pm 4.36$ & $8.20 \pm 5.02(\mathrm{p}<0.001)^{*}$ & $4.79 \pm 3.43(\mathrm{p}<0.001)^{*}$ & $3.36 \pm 3.06(\mathrm{p}<0.001)^{*}$ \\
- Storage score & $7.14 \pm 2.59$ & $4.43 \pm 2.60(\mathrm{p}<0.001)^{*}$ & $3.28 \pm 1.18(\mathrm{p}<0.001)^{*}$ & $2.87 \pm 1.95(\mathrm{p}<0.001)^{*}$ \\
IPSS-QOL & $5.07 \pm 0.39$ & $2.56 \pm 1.63(\mathrm{p}<0.001)^{*}$ & $1.51 \pm 1.07(\mathrm{p}<0.001)^{*}$ & $1.20 \pm 0.82(\mathrm{p}<0.001)^{*}$ \\
NQOL (1-13) & $32.10 \pm 9.17$ & $35.67 \pm 8.29(\mathrm{p}<0.004)^{*}$ & $39.31 \pm 5.51(\mathrm{p}<0.001)^{*}$ & $42.89 \pm 5.25(\mathrm{p}<0.001)^{*}$ \\
IIEF & $11.61 \pm 6.69$ & $11.63 \pm 7.41$ & $11.43 \pm 7.11$ & $11.93 \pm 7.42$ \\
\hline
\end{tabular}

$n=49$

Data are presented as mean $\pm S D$

$* p<0.05$ significant, Repeated measurement analysis of variance (ANOVA) 
Table 2 - Number of nocturia episodes and hours of undisturbed sleep (HUS) at baseline and after 4 and 8 weeks of treatment.

\begin{tabular}{lccc}
\hline Variable & Baseline & Visit 4 (Week 4) & Visit 5 (Week 8) \\
\hline Nocturia episode & $3.12 \pm 0.40$ & $1.90 \pm 0.83(\mathrm{p}<0.001)^{*}$ & $1.72 \pm 0.88(\mathrm{p}<0.001)^{*}$ \\
HUS (hours) & $2.67 \pm 1.13$ & $3.02 \pm 1.12$ & $3.25 \pm 1.51(\mathrm{p}=0.010)^{*}$ \\
\hline
\end{tabular}

$n=49$

Data are presented as mean $\pm S D$

$* p<0.05$ significant, Repeated measures analysis of variance (ANOVA)

Table 3 - Uroflowmetry outcomes at baseline and after 2, 4 and 8 weeks of treatment.

\begin{tabular}{lcccc}
\hline Variable & Baseline & Visit 3 (Week 2) & Visit 4 (Week 4) & Visit 5 (Week 8) \\
\hline $\mathbf{Q}_{\max }(\mathbf{m L} / \mathbf{s})$ & $11.04 \pm 4.07$ & $13.16 \pm 6.17(\mathrm{p}=0.004)^{*}$ & $13.03 \pm 5.4(\mathrm{p}=0.003)^{*}$ & $13.70 \pm 5.87(\mathrm{p}<0.001)^{*}$ \\
$\mathbf{Q}_{\text {ave }}(\mathbf{m L} / \mathbf{s})$ & $5.51 \pm 2.42$ & $6.46 \pm 2.97(\mathrm{p}=0.017)^{*}$ & $7.56 \pm 5.52(\mathrm{p}=0.002)^{*}$ & $7.05 \pm 3.61(\mathrm{p}<0.001)^{*}$ \\
Void urine & $239.40 \pm 118.54$ & $244.85 \pm 130.72$ & $253.89 \pm 120.37$ & $280.00 \pm 131.17$ \\
volume $(\mathbf{m L})$ & & & & $46.77 \pm 19.68$ \\
Void time (sec) & $54.45 \pm 27.73$ & $47.36 \pm 25.05$ & $13.95 \pm 13.05$ & $12.45 \pm 8.16$ \\
Time to $\mathbf{Q}_{\max }$ & $10.62 \pm 8.65$ & $14.35 \pm 10.96$ & & \\
$(\mathbf{s e c})$ & & & & \\
\hline
\end{tabular}

$n=49$

Data are presented as mean $\pm S D$

$* p<0.05$ significant, Repeated measures analysis of variance (ANOVA)

was observed in $20(40.82 \%), 22(44.90 \%)$ and 26 (53.06\%) patients at visits 3 (week 2), 4 (week 4) and 5 (week 8), respectively.

\section{Safety}

Blood pressure, heart rate and blood chemistry results at the last visit were not significantly different from baseline values. Three patients $(5.88 \%)$ out of the total 51 experienced mild dizziness, but all patients tolerated the medication during the treatment period.

\section{DISCUSSION}

The alpha adrenergic receptor antagonists have evolved from non-selective antagonists to short acting selective and then long acting selective and subtype selective antagonists. The alpha 1a, and to a lesser extent alpha $1 \mathrm{~d}$, receptors predominate in urologic tissues, whereas the alpha $1 \mathrm{~b}$ receptors are found elsewhere, such as the vascular tissues $(6,7)$. The ideal alpha receptor antagonist should be uroselective with minimal side effects. Tamsulosin shows ten times higher affinity for alpha $1 \mathrm{a}$ than alpha $1 \mathrm{~b}$ receptors (79).

Tamsulosin is a superselective subtype alpha 1a and 1d blocker for the treatment of LUTS associated with $\mathrm{BPH}$. The standard dose of tamsulosin for $\mathrm{BPH}$ is $0.4 \mathrm{mg}(8)$ but a lower dose $(0.2 \mathrm{mg})$ is also used in Asia $(10,11)$. The conventional Tamsulosin formulation has an absorption profile that is dependent on food intake, and is associated with low levels of intestinal absorption. The serum level is dependent on food and gastrointestinal transit time, resulting in $70 \%$ higher serum levels when taken on an empty stomach compared to after a meal (12). The new formulation, Tamsulosin OCAS $\AA$, which contains $0.4 \mathrm{mg}$ active Tamsulosin hydrochloride, was designed to improve drug delivery compared to the previous formulations. The OCAS ${ }^{\circledR}$ formulation is a gel matrix comprising gel-forming and gel-enhancing agents $(2,3)$. Following administration, the Tamsulosin OCAS ${ }^{\circledR}$ tablet 
Figure 2 - Mean IPSS scores before and after treatment.

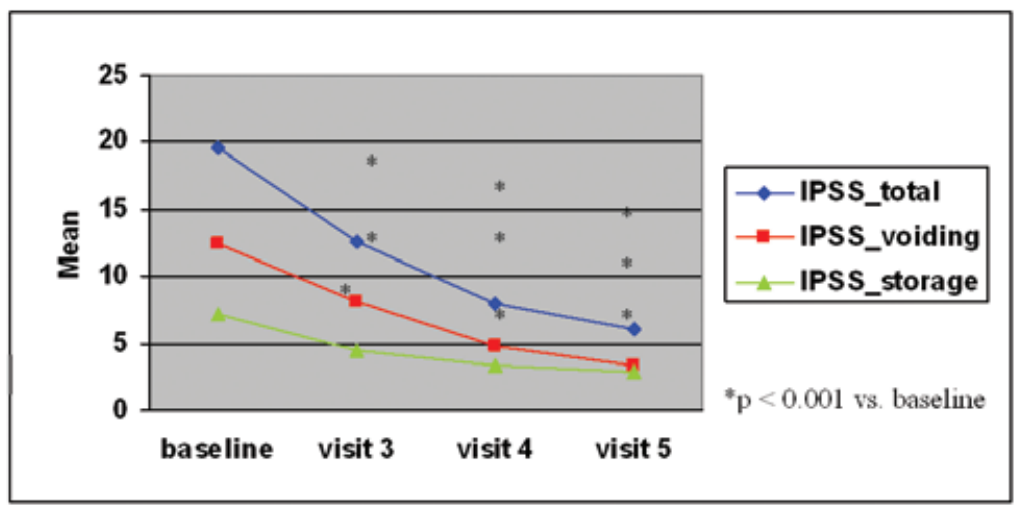

IPSS, International Prostate Symptom Score

Figure 3 - Improvement in IPSS-QoL.

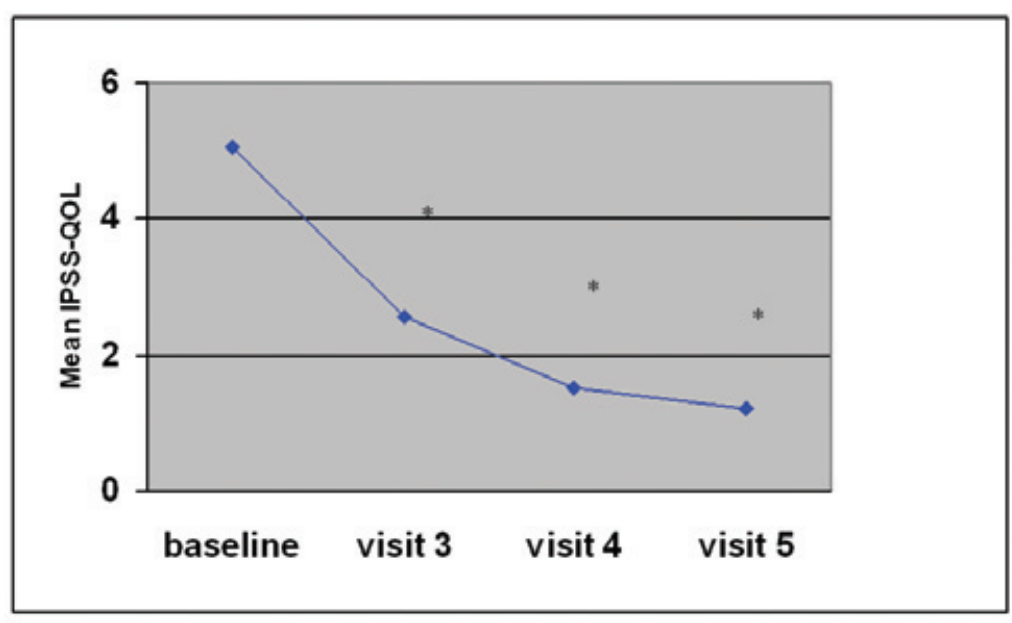

IPSS-QOL, International Prostate Symptom Score Quality Of Life

Figure 4 -Improvement in N-QOL (Items 1-13).

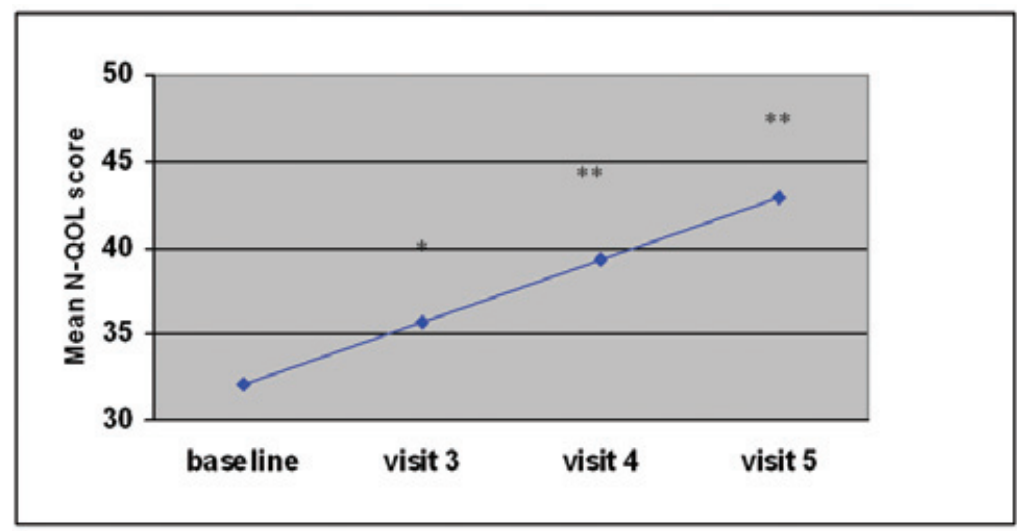

${ }^{*} \mathrm{p}<0.004$ vs. baseline

${ }^{* *} \mathrm{p}<0.001$ vs. baseline

N-QoL, Nocturia Quality Of Life 
undergoes rapid gelation and complete hydration. The tablet carries water throughout the entire gastrointestinal tract, including the colon, where water content is lowest, resulting in consistent drug release independent of $\mathrm{pH}$ and gastrointestinal motility $(2,3)$. Thus, Tamsulosin OCAS ${ }^{\circledR}$ has a superior pharmacokinetic profile than conventional Tamsulosin, allowing it to deliver consistent drug levels over 24 hours with lower maximum plasma concentration $\left(\mathrm{C}_{\max }\right)$, irrespective of food intake. The 24-hour sustained efficacy extending over a longer period of time, even at night, leads to improvements in LUTS/BPH, such as nocturia with fewer side effects. This effect can be explained by the specific mechanism of alpha blockers (13).

Nocturia is one of the most bothersome LUTS in BPH, and is the most common cause of sleep disturbance in older men. The prevalence of nocturia as a cause of disturbed sleep increases with age. Nocturia and disturbed sleep are associated with a range of serious co-morbid conditions, increased risk for accidents at home and on the road, reduced daytime alertness and activity, reduced sense of wellbeing, depression, mood change, and increased disease susceptibility and mortality (11).

Most of the restorative sleep occurs in the first half of the night. Therefore, it is important to avoid awakening during the first few hours of sleep. For good quality sleep, the HUS should be at least 3 to 4 hours. Increasing the HUS leads to improved QoL. Tamsulosin OCAS $®$ represented a statistically significant $57 \%$ improvement in nocturia control (14).

Alpha blockers not only inhibit alpha 1-adrenergic receptors in the prostate and bladder neck, but also have an effect on the cardiovascular system. The randomized crossover study concluded that Tamsulosin OCAS ${ }^{\circledR}$ had a lower incidence of positive stress test than conventional Tamsulosin (15).

Our study demonstrated that tamsulosin OCAS ${ }^{\circledR} 0.4 \mathrm{mg}$ improves the IPSS total and subscores, nocturia, HUS and QoL in BPH patients. We use N-QoL questionnaires for evaluation of QoL, it is the self- administered questionnaire that has been proven to correlate with the energy/ vitality domains of the SF -36 questionnaire and with the sleep quality of Pittsburgh Sleep Quality Index (16). Total IPSS significantly decreased at week 8 from baseline (from 19.52 to $6.08 ; \mathrm{p}<0.001)$. Similarly, the voiding and storage scores of IPSS also continued to improve significantly starting from the second and third visits, respectively ( $p<0.001$ versus baseline). The IPSSQoL and N-QoL scores significantly improved at visit 3 throughout the study. In addition, significant improvements also occurred in nocturia episodes and HUS at the last visit. Uroflowmetry parameters, $Q_{\max }$ and $\mathrm{Q}_{\text {ave }}$, improved significantly at visit 3 through end of study. The response to treatment in these patients was evident within 2 weeks after treatment. All patients had at least a $25 \%$ improvement in total IPSS score versus baseline, and $97.96 \%$ had more than a 1-point decrease in IPSS-QOL score and, therefore, can be considered responders of the treatment.

This study demonstrated the favourable cardiovascular safety profile of Tamsulosin OCAS ${ }^{\circledR}$ which helped BPH patients with cardiovascular comorbidities and are taking antihypertensive medications. Most patients with lower BMI generally tolerate Tamsulosin OCAS ${ }^{\circledR}$ very well with only few side effects, which are usually mild and all patients tolerated the medication during the treatment period. The specific pharmacokinetic properties of Tamsulosin OCAS ${ }^{\circledR}$ with its efficacy and safety profile in $\mathrm{BPH}$ patients provided the desirable therapeutic risk benefit ratio.

The limitations of this study are: 1) lack of control group either with placebo or with traditional Tamsulosin, 2) lack of urodynamic assessment preoperatively and postoperatively, 3) under powered patient population. This is the preliminary report with small number of patients that will require larger cohort study in the future study.

\section{CONCLUSIONS}

Alpha adrenergic antagonists are the first line treatment for LUTS due to BPH. Tamsulosin OCAS ${ }^{\circledR}$ rapidly improves LUTS, both voiding and storage symptoms. The specific pharmacokinetic profile of Tamsulosin OCAS ${ }^{\circledR}$ provides an effective treatment for nocturia and HUS with less side effects. Tamsulosin OCAS ${ }^{\circledR}$ also improves QoL in patients with LUTS due to BOO associated with BPH. 


\section{CONFLICT OF INTEREST}

None declared.

\section{ACKNOWLEDGEMENTS}

We thank Ms Chichanok Ruengorn and Ms Wilaiwan Chongruksut for the statistical analysis.

\section{REFERENCES}

1. van Kerrebroeck P, Abrams P, Chaikin D, Donovan J, Fonda D, Jackson S, et al.bThe standardisation of terminology in nocturia: report from the Standardisation Sub-committee of the International Continence Society. Neurourol Urodyn. 2002; 21: 179-83.

2. Franco-Salinas G, de la Rosette JJ, Michel MC. Pharmacokinetics and pharmacodynamics of tamsulosin in its modified-release and oral controlled absorption system formulations. Clin Pharmacokinet. 2010; 49: 17788.

3. Nargund VH, Grey AD. Tamsulosin MR and OCAS (modified release and oral controlled absorption system): current therapeutic uses. Expert Opin Pharmacother. 2008; 9: 813-24.

4. Chapple CR, Chartier-Kastler E. Pharmacokinetic profile of tamsulosin OCAS. BJU Int. 2006; 98: S9-S12.

5. Neill MG, Shahani R, Zlotta AR. Tamsulosin oral controlled absorption system (OCAS) in the treatment of benign prostatic hypertrophy. Ther Clin Risk Manag. 2008; 4: 11-8.

6. Lepor H. Role of alpha-adrenergic blockers in the treatment of benign prostatic hyperplasia. Prostate Suppl. 1990; $3: 75-84$.

7. Kenny BA, Miller AM, Williamson IJ, O'Connell J, Chalmers DH, Naylor AM. Evaluation of the pharmacological selectivity profile of alpha 1 adrenoceptor antagonists at prostatic alpha 1 adrenoceptors: binding, functional and in vivo studies. Br J Pharmacol. 1996; 118: 871-8

8. Roehrborn CG, Schwinn DA. Alpha1-adrenergic receptors and their inhibitors in lower urinary tract symptoms and benign prostatic hyperplasia. J Urol. 2004; 171: 1029-35.

9. Roehrborn CG, Siegel RL. Safety and efficacy of doxazosin in benign prostatic hyperplasia: a pooled analy- sis of three double-blind, placebo-controlled studies. Urology. 1996; 48: 406-15.

10. Li NC, Chen S, Yang XH, Du LD, Wang JY, Na YQ, et al. Efficacy of low-dose tamsulosin in chinese patients with symptomatic benign prostatic hyperplasia. Clin Drug Investig. 2003; 23: 781-7.

11. Park CH, Chang HS, Oh BR, Kim HJ, Sul CK, Chung SK, et al. Efficacy of low-dose tamsulosin on lower urinary tract symptoms suggestive of benign prostatic hyperplasia: a nonblind multicentre korean study. Clin Drug Investig. 2004; 24: 41-7.

12. Lyseng-Williamson KA, Jarvis B, Wagstaff AJ. Tamsulosin: an update of its role in the management of lower urinary tract symptoms. Drugs. 2002; 62: 135-67.

13. Koritsiadis G, Tyritzis SI, Koutalellis G, Lazaris AC, Stravodimos K. The effect of alpha-blocker treatment on bladder hypoxia inducible factor-1 alpha regulation during lower urinary tract obstruction. Int Braz J Urol. 2010; 36: 86-94.

14. Stanley N. The physiology of sleep and the impact of ageing. Eur Urol Suppl. 2005; 3: 17-23.

15. Michel MC, Korstanje C, Krauwinkel W, Shear M, Davies J, Quartel A. Cardiovascular safety of the oral controlled absorption system (OCAS) formulation of tamsulosin compared to the Modified release (MR) formulation. Eur Urol Suppl. 2005; 4: 53-60.

16. Buysse DJ, Reynolds CF 3rd, Monk TH, Berman SR, Kupfer DJ. The Pittsburgh Sleep Quality Index: a new instrument for psychiatric practice and research. Psychiatry Res. 1989; 28: 193-213.

\section{Submitted for publication:} July 19, 2010

Accepted after revision:

December 21, 2010

\footnotetext{
Correspondence address:

Dr. Bannakij Lojanapiwat

Division of Urology, Department of Surgery

Chiangmai University, Chiangmai, 50200, Thailand

Fax: + 6653945154

E-mail: blojanap@mail.med.cmu.ac.th
} 